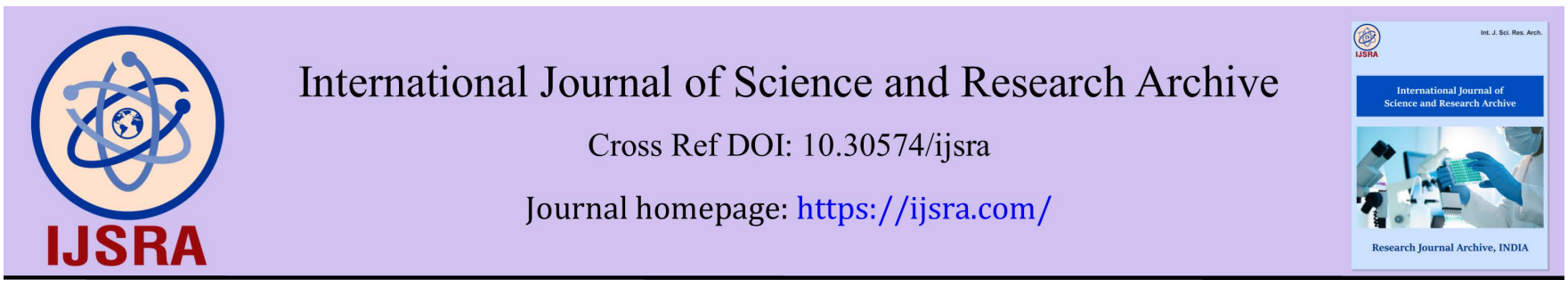

(RESEARCH ARTICLE)

\title{
Phyllodic tumors of the breast: Frequency and anatomo-histoclinical aspects at Conakry University Teaching Hospital (CHU). Guinea
}

Boubacar siddi Diallo 1,* , Boubacar alpha Diallo 1, Ibrahima conte ${ }^{2}$, Abdoulaye sylla ${ }^{3}$, Diallo yaya ${ }^{2}$, Diallo m.k ${ }^{3}$,Ibrahima sory balde ${ }^{2}$, moussa koulibaly ${ }^{3}$, telly sy ${ }^{2}$, yolande hyjazi ${ }^{1}$ and namory keita ${ }^{1}$

${ }^{1}$ University Department of Gynecology Obstetrics, Donka National Hospital, Conakry Guinea.

2 University Department of Gynecology Obstetrics, Ignace Deen National Hospital, Conakry Guinea.

${ }^{3}$ University Department of Anatomy Pathology, Donka National Hospital, Conakry Guinea.

International Journal of Science and Research Archive, 2021, 02(01), 001-006

Publication history: Received on 09 January 2021; revised on 17 January 2021; accepted on 19 January 2021

Article DOI: https://doi.org/10.30574/ijsra.2021.2.1.0009

\begin{abstract}
Objectives: Calculate the frequency of phyllodes tumors of the breast, describe the epidemiological profile and describe the anatomo-histoclinical aspects of phyllodes tumors of the breast at the Conakry University Teaching Hospital.

Methodology: This was a retrospective descriptive type study lasting 10 years, from January 1, 2008 to December 31, 2018. It concerned all cases of breast lesions, the parts that were examined in the anatomo-pathological laboratory. All cases of mammary tumours were included for the diagnosis of phyllodes tumor which was histologically confirmed. All cases of breast lesions for which the diagnosis was histologically ruled out were excluded. We carried out an exhaustive examination of the data available in the registers of the anatomo-pathologies service of the Conakry University Teaching Hospital. . The limitations or constraints of the study were the absence of certain information on the pathological examination request forms and the absence of an immunohistochemical study.
\end{abstract}

Results: The frequency of phyllodes tumors was $02.72 \%(\mathrm{n}=22)$ among benign and malignant breast pathologies $(\mathrm{n}=$ $807)$ and $1.65 \%$ among those of genital and breast pathologies $(n=1334)$. The epidemiologic profile was that of a woman in the age group 50-59 (27.27\%), nulliparous (45.45\%). Inflammation was the main reason for consultation $(100 \%)$. The main presumptive clinical diagnosis was benign tumor (54.54\%). Lumpectomy was the main surgical procedure (36.36\%) followed by excised biopsies (27.27\%). Cases of phyllodes with a consistency firm were the most observed (50\%) followed by cases with associated consistency 6 cases $(27.27 \%)$. Phyllodes tumours without rearrangements represented (63.63\%) followed by cases with associated rearrangements (13.63\%). Grade I constituted the majority of the histological grade found (54.54\%) followed by grade II (22.73\%). Phyllodes tumors with benign behavior constituted the majority of cases observed 17 (77.27\%). Tumors resected in a healthy zone were the most represented $(72.73 \%)$. The histologic types with good prognosis constituted the majority of our diagnosed cases (77.27\%).

Conclusion: Phyllodes tumours constitute of a particular histological type of fibroepithelial tumours of the breast. They constitute a predominantly benign tumor, its malignant forms consist of high-grade sarcomas, often with a poor prognosis. They affect all ages, but especially women between the third and fifth decade. Their diagnosis is exclusively histological, which makes it possible to assess to their behavior, grade and histological prognosis

Keywords: Breast; Phyllode; Frequency.

\footnotetext{
* Corresponding author: Docteur Diallo Boubacar Siddi

University Department of Gynecology Obstetrics , Donka national Hospital , Conakry Guinea.
} 


\section{Introduction}

The phyllodes tumors constitute of mammary tumors with double differentiation, which can have histological characters of benignity or of malignancy according to the histological grades. [1]

Many of its forms are benign, but the malignant forms constitute high grade sarcomas. They are distributed worldwide, affecting all ages, but with a predominance in women in full genital activity between the 3rd and 5th decades. They affect all races but, with a predominance among black women. Their frequency is estimated to be approximately 0.3 to $0.9 \%$ of primary breast tumors, according to Ngou Mwe Ngou et al [2] the etiopathogenesis of phyllodes tumors remains unknown [3]. The histology remains the essential and fundamental time for the diagnosis of phyllodes tumors, specifying their grade and their histopronosis. The problem that generally arises is to differentiate histologically a grade 4 phyllodes tumor from primary angiosarcoma or fibrohistiocytic sarcoma or breast fibrosarcoma, which is of interest in some cases to the immunohistochemical study. [4].

Their prognosis is generally based on grade, surgical section and necrosis. They represent a tumor with very high recurrence and / or high metastatic potential, in particular pulmonary. Surgery constitutes the radical treatment of these tumors [1]. Malignant forms associating loco regional or distant metastases, would require radiotherapy and associated surgery [5]. The objectives of this study were to calculate the frequency of phyllodes tumors of the breast, describe the epidemiological profile and describe the anatomo-histoclinical aspects of phyllodes tumors of the breast at the Conakry University Teaching Hospital.

\section{Methodology}

This was a descriptive retrospective study lasting 10 years, from January 1, 2008 to December 31, 2018. It concerned all cases of breast lesions, the parts that were examined in the anatomo pathological laboratory. All cases of mammary tumors for which the diagnosis of phyllodes tumor was confirmed histologically were included. All cases of breast lesions for which the diagnosis was histologically ruled out were excluded. We carried out an exhaustive examination of the data available in the registers of the anatomo-pathologies service of the Conakry University Teaching Hospital. The limitations or constraints of the study were the absence of certain information on the pathological examination request forms and the absence of an immunohistochemical study. The variables studied were epidemiological (frequency, age, parity), clinical (reasons for consultation and presumptive clinical diagnosis, type of sample) and anatomopathological: macroscopic (consistency of the lesion, associated changes) histological (histological grade, behavior of tumor, histo-prognosis).

\section{Results}

\subsection{Frequency}

The frequency of phyllodes tumors was $02.72 \%(n=22)$ among benign and malignant breast pathologies $(n=807)$ and $1.65 \%$ among those of genital and breast pathologies $(n=1334)$

\begin{tabular}{|l|l|l|}
\hline Tumor & Number & $\mathbf{\%}$ \\
\hline Phyllodes tumor & 22 & 2,72 \\
\hline Benign breast tumor & 302 & 37,42 \\
\hline Malignant breast tumor & 346 & 42,87 \\
\hline Inflammatory lesions or breast dystrophy & 137 & 16,97 \\
\hline Total & 807 & 100 \\
\hline
\end{tabular}

\subsection{The epidemiological profile}

\subsubsection{Age}

The age group of 50-59 years was the most concerned (27.27\%), followed by that of 40-49 years $(22,72 \%)$. The average age was 44.5 years with extremes of 18 and 83. 


\subsubsection{Parity}

The nulliparas constituted the majority of the cases observed (45.45\%).

\subsection{Clinic}

\subsubsection{Reasons for consultation}

Inflammation was the main reason for consultation (100\%).

\begin{tabular}{|l|l|l|}
\hline Reasons for consultation & Number & $\mathbf{\%}$ \\
\hline Pain & 7 & 31,81 \\
\hline Inflamation & 22 & 100 \\
\hline Gravity & 13 & 59,09 \\
\hline Breast discharge & 1 & 4,54 \\
\hline Ulceration & 1 & 4,54 \\
\hline Total & 22 & 100 \\
\hline
\end{tabular}

\subsubsection{Presumptive clinical diagnosis}

The main presumptive clinical diagnosis was benign tumor (54.54\%).

\subsubsection{The type of sample}

Lumpectomy was the main surgical procedure (36.36\%) followed by excisional biopsies (27.27\%).

\begin{tabular}{|l|l|l|}
\hline Type of sample & Nombre & \% \\
\hline Diagnostic biopsy & 3 & 13,63 \\
\hline Biopsy resection & 6 & 27,27 \\
\hline Quadrantectomy & 2 & 9,09 \\
\hline Tumorectomy & 8 & 36,36 \\
\hline Mastectomy & 1 & 4,54 \\
\hline Zonectomy & 2 & 9,09 \\
\hline Total & 22 & 100 \\
\hline
\end{tabular}

\subsection{Anatomo-pathology:}

\subsubsection{Macroscopy}

- The consistency of the lesion: The cases of phyllodes tumor with firm consistency were the most observed $(50 \%)$ followed by cases with an associated consistency 6 cases $(27.27 \%)$.

- Associated rearrangements: The phyllodes tumors without rearrangements represented (63.63\%) followed by cases with associated rearrangements (13.63\%).

\begin{tabular}{|l|l|l|}
\hline Associated changes & Nombre & $\mathbf{\%}$ \\
\hline Necrotic & 1 & 4,54 \\
\hline Haemorrhagic & 1 & 4,54 \\
\hline Calcium & 2 & 9,09 \\
\hline
\end{tabular}




\begin{tabular}{|l|l|l|}
\hline Chondroid & 1 & 4,54 \\
\hline Not reworking & 14 & 63,63 \\
\hline Associate & 3 & 13,63 \\
\hline Total & 22 & 100 \\
\hline
\end{tabular}

\subsubsection{Histology}

Histological grade

Grade I constituted the majority of the histological grade found ( $54.54 \%)$ followed by grade II, ie (22.73\%).

The behavior of the tumor

Phyllodes tumors with benign behavior constituted the majority of the cases observed $77.27 \%$.

Histo-prognosis

Histological types with good prognosis constituted the majority of our diagnosed cases (77.27\%).

\section{Discussion}

\subsection{Frequency}

The frequency of phyllodes tumors was $02.72 \%(\mathrm{n}=22)$ among benign and malignant breast pathologies $(\mathrm{n}=807)$ and $1.65 \%$ among those of genital and breast pathologies $(\mathrm{n}=1334)$. This observation shows that there are tumor lesions of the breast which can be benign or malignant. These benign forms constitute a histological element of differential diagnosis with the fibroadenoma. They share in common several histological features with the breast fibroadenoma which are fibroepithelial contingent tumors hence the name phyllodes giant fibroadenoma tumors. This result is much higher than those reported by KANOUNI L. et al. [6], Gaétan Mac grogan [7] and ALAMI A et al. [8], who found respectively $0.09 \%, 0.5 \%$ and $1 \%$ phyllodes tumor among primary mammary tumors. We could explain this result by the fact that all these authors carried out their studies in clinics specializing in oncology and breast pathologies practices and who specifically receive these pathologies.

\subsection{The epidemiological profile:}

\subsubsection{Age}

The age group of 50-59 years was the most concerned (27.27\%), followed by that of 40-49 years (22, 72\%). The average age was 44.5 years with extremes of 18 and 83. Our study shows that phyllodes tumors mainly affect older women, especially between the 4th and 6th decades, but all age groups can be affected. This result is superimposable on that reported by Gaetan Mcgrogan [7], ie an average age of 45 years and significantly higher than those reported by Kanouni L et Coll. [6] and Sabban. F et al. [9] who found a mean age of 30 and 33.4 years, respectively.

\subsubsection{Parity}

The nulliparas constituted the majority of the cases observed (45.45\%). This result can be superimposed on that found by Kanouni. L et al. [6] ie $44.4 \%$ in nulliparous but clearly inferior to Sabban's result. F et al. [9] or $62.5 \%$.

\subsubsection{Clinic}

\section{Reasons for consultation}

Inflammation was the main reason for consultation (100\%). Alami. L et al. [8] reported in their series that tumor mass and mammary deformity constitute the main reason for consultation or the main circumstance of discovery of phyllodes tumors and that the pain generally reflects nerve compression secondary to a malignant transformation of these tumors. Gaétan Macgrogan [7] in his series indicates that the phyllodes tumor appears clinically in the form of a palpable nodule that nothing distinguishes clinically a banal fibroadenoma and that sometimes, there is a notion of rapid growth from Novo or from a nodule known for several years. 
Presumptive clinical diagnosis

The main presumptive clinical diagnosis was benign tumor (54.54\%).

The type of sample

Lumpectomy was the main surgical procedure (36.36\%) followed by excisional biopsies (27.27\%). This result is much lower than that found by Sabban. F et al. [10] ie 87.5\% lumpectomy and close to that of Matar. N et al. [10] who report $44 \%$ of enlarged lumpectomy and (29.2\%) of simple mastectomy.

\subsection{Anatomo-pathology}

\subsubsection{Macroscopy}

- $\quad$ The consistency of the lesion: The cases of phyllodes tumor of firm consistency were the most observed (50\%) followed by cases of associated consistency 6 cases $(27.27 \%)$. This observation shows that phyllodes tumors are solid tumors which are more or less resistant to digital pressure. But this criterion of the firmness of a phyllodes tumor is not a significant criterion for assessing the benignity or malignancy of phyllodes tumors. This observation matches that of Leveque. J et al. [3] who report that phyllodes tumors are generally 53\% firm consistency tumors, especially for benign grade 1 and 2 forms, but sometimes their consistency can be hard for forms of malignant transformation (sarcoma).

- Associated rearrangements: The phyllodes tumors without rearrangements represented (63.63\%) followed by cases with associated rearrangements (13.63\%).

This observation is similar to that of El Hanchi. Z et al. [11] or 65\% who reported in their edition that phyllodes tumors are generally homogeneous tumors not accompanied by necrotic, hemorrhagic or calcium changes. The presence of rearrangements within a phyllodes tumor is characteristic of a malignant transformation of the tumor.

\subsubsection{Histology}

\section{Histological grade}

Grade I constituted the majority of the histological grade found (54.54\%) followed by grade II, ie (22.73\%). This observation is classic, because the grades constitute the main criterion of the histopronosis of phyllodes tumors (grade I and II phyllodes tumors are considered as tumors with benign behavior, grades III and IV as those with malignant behavior) which is close to that found by Matar. N et Coll [10] who report $65.5 \%$ of grade I and II; $9.8 \%$ grade III and $17.1 \%$ grade IV.

\section{The behavior of the tumor}

Phyllodes tumors with benign behavior constituted the majority of the cases observed 77.27\%. This observation shows that they are mostly benign, but their course is unpredictable. The malignant forms constitute high-grade sarcomas with unpredictable evolution. This observation is classic and close to those found by Grenier. J et al. [12] and AMY D. [13] who report $65 \%$ and $70 \%$ respectively with benign behavior. The same authors report that the differential diagnosis between phyllodes tumor and fibroadenoma is not always easy, it requires careful histological analyzes.

\section{Histological prognosis}

Histological types with a good prognosis constituted the majority of our diagnosed cases (77.27\%). This observation is very illustrative although the phyllodes tumors with a good prognosis have been largely in the majority, it requires careful monitoring because a phyllodes tumor with a good prognosis can undergo sarcomatous transformation. M. A et al. [14] reported in their series that grade is an element of histopronostic, but the surgical section is the major histopronostic element. For their part, Grenier J et Coll. [12] report in their series that the histoprognosis of phyllodes tumors is based on grade, involvement of the resection margins and sometimes tumor necrosis. But the most important criterion is the tumor resection margin. Residual tumours should be resected with a very wide resection margin and thoroughly investigated in the pathology laboratory. Because each residual phyllodes tumor has a very high recurrence potential and a risk of significant sarcomatous transformation.

\section{Conclusion}

Phyllodes tumors constitute a particular histological type of fibroepithelial tumors of the breast. They constitute a predominantly benign tumor, its malignant forms consist of high-grade sarcomas, often with a poor prognosis. They 
affect all ages, but especially women between the third and fifth decade. Their diagnosis is exclusively histological, which makes it possible to assess their behavior, grade and histological prognosis.

\section{Compliance with ethical standards}

\section{Statement of informed consent}

"Informed consent was obtained from all individual participants included in the study."

\section{References}

[1] Cabaret V. Delobelle-Deroide A. Vilain MO. Phyllodes tumors. Arch Anat Cytol Pathol. 1995; 43: 59-72.

[2] Large PH, Morcillo JP, Crenn Y, Charles G, Meyran M. Epidemiology and pathological anatomy of primary breast cancers; Med. Arm. 1993; 21: 1.

[3] Leveque J.; Wattier E.; Paumier V. Phyllodes tumors in adolescents: Benefits of multidisciplinary management. The breast (Paris). 1996; 6(1): 33-36.

[4] Ngou Mwe Ngou JP, Mayi-Tsonga S, Boukanga NP. Phyllodes tumors of the breast in Libreville: Epidemiology and prognosis. The breast (Paris). 2005; 15(1-2): 32-35.

[5] Defechereux T, Hamoir E, Meurisse M. The mammary phyllodes tumor. RMLG. Medical review of Liège A. 2002; 57(10): 291-297.

[6] Kanouni L, Saadi I. Management of phyllodes tumors of the breast at the National Institute of Oncology in Rabat. Obstetrics \& Fertility Gynecology A. 2004; 32(4): 293-301.

[7] Gaetan Macgrogan. Diagnostic pitfalls in mammary pathology. Case $\mathrm{n}^{\circ}$ 3. Tumor of a fibroepithelial nature with hyper-cellular stroma suggesting a phyllodes tumor. Annales de pathologie (Paris) A. 2009; 29(3): 199-204.

[8] Alami A, Bennani A, Hassi Rahou D, Phyllode tumors. Medical expectation A. 2002; 9(79): 36-38.

[9] Sabban F, Collinet P, Lucot JP. Phyllodes tumors of the breast: about 8 patients. Journal of Obstetrics and Gynecology and Reproductive Biology A. 2005; 34(1): 252-256.

[10] Matar n, Soumani A, Noun M. Phyllodes tumors of the breast. About 41 cases. Journal of Obstetrics and Gynecology and Reproductive Biology A. 1997; 26(1): 32-36.

[11] El hachi Z, Berrada R, Fadli A. Phyllodes tumors of the breast: a review of 23 cases. Maghreb medical A. 2003; 367: 139-142.

[12] Grenier J, Delbaldo C, Zelek L. Phyllodes tumors and sarcomas of the breast: update. Cancer Bulletin A. 2010; 97(10): 1197-1207.

[13] Amy D. Breast ultrasound: examination technique, anatomical results and criteria for benignity. JEMU. Journal of Ultrasound and Ultrasound Medicine A. 1995; 16(6): 235-253.

[14] Latteri MA, Cipollac C, Amato C. Phyllodes tumors of the breast. Lyon Surgical A. 1996; 92(2): 105-108. 